\title{
Metacognitive Training at Home: Does It Improve Older Adults' Learning?
}

\author{
Heather Bailey ${ }^{a}$ John Dunlosky ${ }^{a}$ Christopher Hertzog ${ }^{b}$ \\ ${ }^{a}$ Kent State University, Kent, Ohio, ${ }^{b}$ Georgia Institute of Technology, Atlanta, Ga., USA
}

\author{
Key Words \\ Memory intervention · At-home training $\cdot$ Self-testing \\ training method $\cdot$ Metacognitive skills $\cdot$ Memory \\ competence
}

\begin{abstract}
Background: Previous research has described the success of an intervention aimed at improving older adults' ability to regulate their learning. This metacognitive approach involves teaching older adults to allocate their study time more efficiently by testing themselves and restudying items that are less well learned. Objective: Although this type of memory intervention has shown promise, training older adults to test themselves in the laboratory can be very timeintensive. Thus, the purpose of the present study is to transport the self-testing training method from the laboratory to home use. Methods: A standard intervention design was used that included a pretraining session, multiple training sessions, and a posttraining session. Participants were randomly assigned to either the training group $(n=29)$ or the waiting list control group ( $n=27)$. Moreover, we screened participants for whether they used the self-testing strategy during their pretraining test session. Results: Compared to the performance of the control group, the training group displayed significant gains, which demonstrates that older adults can benefit from training themselves to use these
\end{abstract}

skills at home. Moreover, the results of the present study indicate that this metacognitive approach can effectively improve older adults' learning, even in those who spontaneously self-test prior to training. Conclusions: Training metacognitive skills, such as self-testing and efficient study allocation, can improve the ability to learn new information in healthy older adults. More importantly, older adult clients can be supplied with an at-home training manual, which will ease the burden on practitioners.

Copyright $\odot 2009$ S. Karger AG, Basel

A primary goal of memory interventions is to improve older adults' performance on memory tasks, such as serial word recall, free recall, and face-name and list-learning tasks. In their meta-analysis, Verhaeghen et al. [1] revealed that older adults benefit from interventions that include training memory mnemonics, such as using interactive imagery to associate pairs of items. More recently, a large-scale multi-site training study definitively demonstrated that training mnemonics can enhance older adults' memory performance [2].

We focus on a metacognitive intervention based on training self-regulation skills that can augment standard mnemonic training. In particular, Dunlosky et al. [3] introduced an intervention that involves training two interrelated self-regulation strategies: (a) self-testing and

\section{KARGER}

(C) 2009 S. Karger AG, Basel

Fax +4161306 1234

E-Mail karger@karger.ch

www.karger.com
Accessible online at: www.karger.com/ger
Heather Bailey

PO Box 5190, Department of Psychology

Kent State University

Kent, OH 44242-0001 (USA)

Tel. +1 330672 2169, Fax +1 330672 3786, E-Mail hroth@kent.edu 
(b) efficient allocation of study time. Specifically, for selftesting, older adults studied word pairs (e.g. chateau castle) and then later tested their learning by covering the response for each pair, reading the cue alone (e.g. chateau), and then attempting to retrieve the response from memory. Then, they used the outcomes of these self-tests to decide whether and how long to restudy the pairs (i.e. study allocation). If they could not recall the correct response (e.g. castle), they were instructed to set that particular word pair aside for additional study. Thus, this metacognitive intervention involved training two component skills: (a) self-testing to discover which items have (versus have not) been learned, and (b) allocating subsequent study time to less well-learned items. The older adults' memory for the word pairs was assessed by a preand posttraining test. Dunlosky et al. [3] reported that performance gains from pre- to posttest were much greater for older adults trained in self-testing $(\mathrm{d}=0.72)$ than for those in a randomized waiting list control group $(\mathrm{d}=$ $0.03)$.

In the present study, we had two major goals. First, we sought to transport this metacognitive intervention from the laboratory to home use. Importing training programs into home environments is essential. Not only do some older adults have limited time or ability to attend training programs away from their homes, but training these skills requires involvement by trainers who may not always be available or affordable. Thus, widespread dissemination of a training technique will require self-administration by older adults. Fortunately, older adults report a high level of satisfaction with at-home training studies [4], and the effect sizes of studies using at-home manuals have been similar to laboratory training studies [1]. For a recent example, a multifactorial intervention in which older adults completed homework assignments demonstrated a significant training effect for both memory performance and self-efficacy [5]. To explore the efficacy of at-home training, we used a standard intervention design that included a pretraining session, multiple training sessions, and a posttraining session. The training sessions were completed in the home and consisted of assignments from a manual that described the two component skills of the metacognitive intervention: how to monitor performance via self-testing and then how to use it to decide which items to restudy. The manual was packaged with multiple lists of paired-associate items and instructions to help older adults to systematically practice these metacognitive skills across four training sessions in 2 consecutive weeks. The manual and related materials are available from the first author.
Our second goal concerned evaluating the benefits of training a skill that an individual has used prior to exposure to a formal training program. For instance, some older adults have pre-experimental knowledge of effective mnemonic strategies, such as interactive imagery and sentence generation for associative learning [6], which may explain their spontaneous use of these mnemonics [7]. Most important, even though many older adults know about and use these mnemonics spontaneously, they also benefit from memory interventions that train them [1]. The same may apply to metacognitive training that involves training adults to self-test during study. Bottiroli et al. [unpubl. data] had older adults study paired associates (e.g. dog - spoon) for a paired-associate recall test (i.e. dog - ?). Over $50 \%$ of the older adults spontaneously used at least one of the component strategies (self-testing) from this metacognitive intervention, and they substantially outperformed those older adults who did not spontaneously self-test. Thus, a distinct possibility is that individuals who already test themselves while studying will not benefit any more from training that focuses on using this skill. If so, practitioners could prescreen older adults to ensure that the training focuses on only those skills that could benefit. Alternatively, the training itself makes explicit why self-testing can improve learning (because it helps to isolate unlearned items for further study) and also provides practice with two of the component skills involved in metacognition: selftesting and study allocation. Thus, older adults who spontaneously self-test may still benefit from metacognitive training.

We evaluated these possibilities by screening participants during their pretraining test session. After completing the pretraining test, participants were asked to report any strategies they used during the task. If participants reported covering the to-be-remembered word or restudying unlearned items, they were designated as self-testers. In addition to those who did not self-test prior to training, we assessed whether self-testers could benefit from at-home training. Finally, we contrasted selfpaced and experimenter-paced criterion tests. On the self-paced test, participants studied word pairs on flashcards for up to $30 \mathrm{~min}$. The participants determined how long and how many times they studied the word pairs. On the experimenter-paced test, word pairs were presented on a computer screen one time for $5 \mathrm{~s}$ each. The format of the self-paced, but not experimenter-paced, task allowed participants to cover the to-be-remembered word, test their memory for it, and determine whether or not they needed to restudy the word pair. Thus, as compared to the 
Table 1. Demographic information

\begin{tabular}{llllllllr}
\hline Variable & \multicolumn{2}{l}{ Non-testers } & & & \multicolumn{2}{l}{ Testers } \\
\cline { 2 - 3 } & training & control & $\mathrm{t}$ & & training & control & \multicolumn{1}{c}{$\mathrm{t}$} \\
\hline Age & $69.7(1.7)$ & $73.2(1.6)$ & -1.51 & & $68.5(1.4)$ & $68.8(2.0)$ & -0.12 \\
Education & $15.1(0.6)$ & $14.0(0.3)$ & 1.66 & & $14.4(0.9)$ & $13.7(0.6)$ & 0.74 \\
Vocabulary & $19.3(1.8)$ & $21.6(1.6)$ & -0.95 & & $20.4(2.8)$ & $22.1(1.6)$ & -0.55 \\
Letter comparison & $17.4(0.9)$ & $15.5(1.1)$ & 1.36 & & $19.4(1.0)$ & $19.2(0.9)$ & 0.04 \\
Pattern comparison & $31.6(1.8)$ & $29.2(1.5)$ & 1.05 & & $34.3(2.4)$ & $31.5(1.1)$ & 1.16 \\
\hline
\end{tabular}

Age ranged from 60 to 89 years. Education = the total number of years of education; maximum vocabulary score $=36$; maximum letter comparison $=42$, and maximum pattern comparison $=60$. Standard errors for the corresponding means are in parentheses. All $\mathrm{p}$ values $>0.10$.

experimenter-paced task, training gains should be larger for the self-paced task because it better affords the use of the metacognitive training.

\section{Methods}

\section{Participants}

A total of 81 older adults were recruited through a newspaper advertisement in northeast Ohio. Four participants were excluded for reporting either a history of dementia, stroke, medications for memory problems, or previous participation in a memory improvement program during a phone screening process. The remaining 77 participants came to the laboratory for a pretraining session and were randomly assigned to one of two groups (metacognitive training or waiting list control). Of the 77 eligible participants, 21 (training, $\mathrm{n}=12$; control, $\mathrm{n}=9$ ) correctly recalled 35 or more words (out of 40) on the self-paced task. Data from these participants were excluded from further analyses because their performance was at a functional ceiling level, and hence could not benefit from training. After exclusions, a total of 29 older adults (12 females, 97\% Caucasian) in the training group and 27 (15 females, $96 \%$ Caucasian) in the control group participated in this study. Groups did not significantly differ in age, vocabulary knowledge [8], or perceptual speed as measured by letter and pattern comparison [9] (table 1). Each person was paid USD 20 for his/her participation.

\section{Materials}

Two memory tasks were administered during both the preand posttraining sessions. Both tasks involved learning nounnoun pairs and were either self-paced or experimenter-paced. Participants completed two versions of each task, and the order of administration (i.e. version A vs. version B) was counterbalanced across test sessions.

Self-Paced Task

Participants studied 40 word pairs (e.g. jail - coffee) with each pair printed on a separate $3 \times 5$ index card. They were given 30 min to learn as many pairs as possible and told they could study in any manner. After 30 min or less (if they self-terminated study- ing), participants were given a written cued-recall test with no time limit. The cue (e.g. jail - ) was provided for each item, and the participant wrote the corresponding response (e.g. coffee) in the space provided. Once they completed their recall test, participants were asked to report any techniques they used while studying the word pairs. That is, they were asked to write down all of the strategies they had used to help them remember the word pairs. If they reported covering the word or restudying unlearned items, they were classified as self-testers.

\section{Experimenter-Paced Task}

Forty word pairs were presented individually at a fixed rate (5 s per pair) on a personal computer, with no opportunity for restudy. After the final pair was presented, they were given the recall test in the same written format used in the self-paced task.

\section{At-Home Memory Improvement Manual}

The manual contained several lessons teaching the self-testing technique and four homework assignments that were to be completed during a 2-week period. For each assignment, participants studied two lists of paired associates (e.g. jail - coffee) using selftesting. The list lengths increased over the 2 weeks (from 5 up to 40 pairs), and a study time limit was imposed according to the level of difficulty (e.g. 5 min for 10 pairs). Prior to the study session for each list, instructions in the manual explained the steps of self-testing: (1) go through all of the flashcards and try to learn them, (2) go through the cards again and cover the word on the right and test your memory for it, (3) if you correctly recalled the word, set the card aside, but if you did not correctly recall it, place it at the back of the deck, and (4) repeat steps 1-3 until the time runs out or you have correctly recalled all of the words.

Following study, participants turned to the response sheet in which all of the cues (e.g. jail) were listed along with a blank space to the right. They were instructed to write down the responses (e.g. coffee) in the corresponding blank. After recalling the words, participants graded their own answers using the flashcards and recorded the time spent studying each set. To ensure compliance, each participant in the training group received a phone call one week into the training program to check their progress. They also brought their completed assignments to the posttraining session for experimenter evaluation, and all participants complied with the training instructions. 


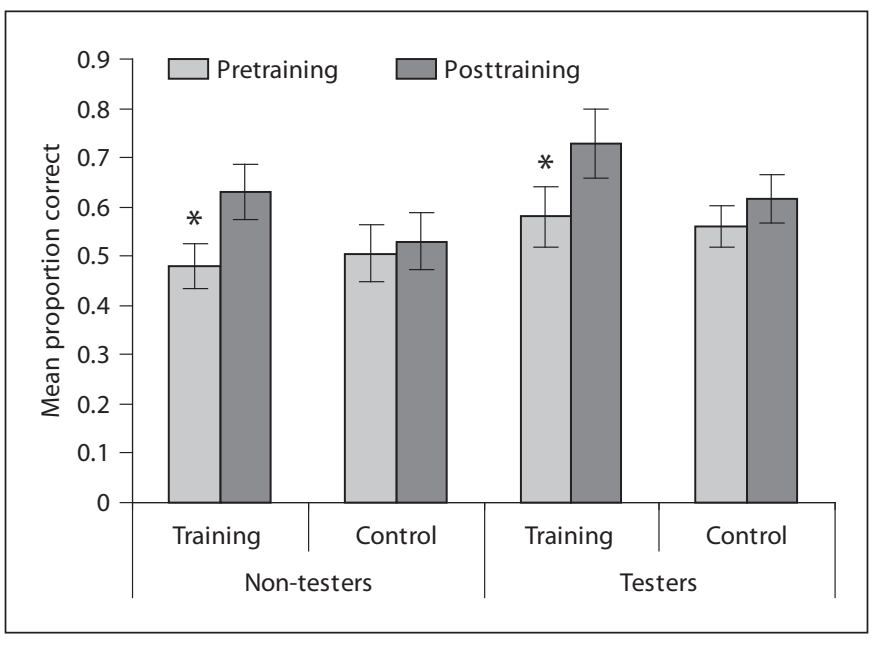

Fig. 1. Mean correct recall on self-paced paired-associate recall task. Asterisk indicates a significant training effect.

Procedure

All participants completed a 1.5 -hour pretraining and a 1hour posttraining test session separated by 2 weeks. The training group worked on the assignments using the at-home manual during the 2-week period, whereas the waiting list control group completed the manual following their posttraining test session.

Participants eligible after the phone screening were brought into the laboratory for the pretraining session. After completing a consent form, participants filled out a demographic questionnaire and underwent measures of vocabulary knowledge [8] and perceptual speed [9]. Next, the participants were given the selfpaced task followed by a global strategy report on which they wrote down all of the strategies they had used to help them remember the word pairs. Finally, participants completed the experimenter-paced task.

Participants came back 2 weeks following their pretraining test session and completed, in this order, the self-paced task, another strategy report, and the experimenter-paced task, in both cases with different lists. Lists were assigned at random to pretest and posttest.

\section{Results}

Ten participants from the training group and ten from the control group reported using at least one of the trained strategies (self-testing and study allocation) during the pretraining session; these participants comprised the tester group. Nineteen participants from the training group and 17 from the control group did not report using either of the two trained skills during the pretraining session; these participants comprised the non-tester group. Although the non-tester group did not use one of the target skills during the pretraining test, they did report using other strategies. Specifically, $20 \%$ of the non-testers reportedly used rote repetition, $20 \%$ used imagery, $26 \%$ generated sentences, and $34 \%$ used some other form of word association. The implication of using these mnemonic strategies (especially effective ones, such as imagery) is that doing so may diminish any benefits that the tester group may gain from using the metacognitive skills. That is, whereas the tester group may benefit from using metacognitive skills, the non-tester group may benefit from using effective mnemonic strategies. This possibility seems unlikely given that the tester group also reported using a similar pattern of mnemonic strategies: $20 \%$ reportedly used rote repetition, $15 \%$ used imagery, $25 \%$ generated sentences, and $40 \%$ used word association. The reported amount of mnemonic strategy use did not differ significantly between the two groups (Mann-Whitney $\mathrm{U}>332.5, \mathrm{p}>0.65$ ), which suggests that both groups were equally likely to use standard mnemonic strategies.

Furthermore, participants from the tester and nontester groups did not differ significantly on a variety of demographic variables. As shown in table 1, participants in each group did not differ in age, processing speed, vocabulary, or years of education, all $\mathrm{p}$ values $>0.10$.

\section{Self-Paced and Experimenter-Paced Recall}

To evaluate the a priori predictions, we conducted a 2 (intervention group: training versus control) $\times 2$ (tester: non-tester versus tester) $\times 2$ (session: pretraining versus posttraining) mixed analysis of variance separately for the self-paced task and experimenter-paced task. We also tested the homogeneity of variances assumption given the smaller sample size in the tester group. The Levene test for equality of variances was not significant either on the self-paced task, $\mathrm{F}(1,54)=0.34, \mathrm{p}=0.57$, or on the experimenter-paced task, $F(1,54)=0.41, p=0.53$, indicating the variances in the tester and non-tester groups are acceptably homogeneous.

For the self-paced task, the mean proportion of correctly recalled items is shown in figure 1 . The expected intervention group $\times$ session interaction was significant, $\mathrm{F}(1,53)=4.34, \mathrm{p}<0.05, \eta^{2}=0.06$, demonstrating that manual-based training in the home produced gains in memory performance from pre- to posttraining sessions beyond that expected by practice effects (i.e. the gains demonstrated by the control group). Although performance tended to be greater overall for testers than nontesters, the main effect for testers only approached significance, $\mathrm{F}(1,53)=2.45, \mathrm{p}=0.12$. The tester $\times$ session 
interaction was not significant, $\mathrm{F}<1$. Both the non-tester group $(\mathrm{d}=0.62[10])$ and the tester group $(\mathrm{d}=0.66)$ benefited from training.

The experimenter-paced task does not afford the use of the self-testing strategy, so we predicted no training gains on this task. The tester $\times$ session interaction was not significant, $\mathrm{F}<1$, so we present the mean values collapsed across the tester group. Participants in the training group correctly recalled $3.8(\mathrm{SEM}=0.6)$ items during their pretraining test and $4.9(\mathrm{SEM}=0.6)$ items during their posttraining test. Participants in the control group correctly recalled $3.3(\mathrm{SEM}=0.7)$ items during their pretraining test and $4.6(\mathrm{SEM}=0.7)$ items during their posttraining test. As predicted, the intervention group $\times$ session interaction was not significant, $\mathrm{F}<1$, indicating that performance on the experimenter-paced task was not influenced by training.

\section{Study Times}

For the self-paced task, a 30-min time limit was imposed, but some participants ended the study session indicating they were ready for the recall test (control $=1$, training $=7$ at pretraining; control $=0$, training $=4$ at posttraining). Mean study time significantly increased for the non-testers in the training group from $28.2 \mathrm{~min}$ $(\mathrm{SEM}=0.9)$ during the pretraining test to $29.8 \mathrm{~min}$ $(\mathrm{SEM}=0.2)$ during the posttraining test, $\mathrm{t}(18)=1.84, \mathrm{p}>$ $0.05, d=0.60$. Study time for the self-testers in the training group did not change significantly from pre- to posttraining sessions. Their mean study time was $29.4 \mathrm{~min}$ $(\mathrm{SEM}=0.6)$ during the pretraining test and $29.6 \mathrm{~min}$ $(\mathrm{SEM}=0.3)$ during the posttraining test. Study time for participants in the waiting list control group also did not change from pre- to posttraining tests, $\mathrm{t}<1.0$. On average, non-testers in the control group spent $29.9 \mathrm{~min}$ (SEM $=0.1$ ) studying during the pretraining test and 30 $\min (\mathrm{SEM}=0.0)$ during the posttraining test, and all of the testers spent the entire 30 min during both tests.

\section{Discussion}

In the current study, we had two goals: to evaluate the efficacy of an at-home metacognitive intervention and to examine these effects after prescreening for those who were already using the to-be-trained strategy. Concerning the former goal, the results provide further support for the benefits of training the component metacognitive skills involved in study, while demonstrating the success of at-home training. In fact, the effect size for metacogni- tive training completed by older adults at home $(d=0.63$; collapsed across testing groups) was nearly as large as the effect size when training was conducted in the laboratory $(\mathrm{d}=0.72[3])$. Thus, practitioners can promote self-regulated learning by supplying older adult clients with an easy-to-use training manual, which is available from any of the authors. Moreover, these metacognitive skills can easily supplement other mnemonic interventions, as demonstrated by Dunlosky et al. [3]. In this study, larger gains were enjoyed by a group of older adults who received mnemonic strategy training (e.g. using interactive imagery) and metacognitive training than by a group who received mnemonic strategy training alone.

Concerning the second goal, participants benefited from the metacognitive intervention regardless of whether they initially reported using self-testing or not. One reason is that even when some older adults report using self-testing or study allocation to regulate their learning, they may not use this strategy consistently across all items without training. After training, they may simply use these metacognitive skills more often across the list. An analogous situation was reported by Dunlosky and Hertzog [7] who reported that (a) uninstructed older adults sometimes used mnemonic strategies (e.g. interactive imagery) to study paired associates, yet (b) older adults who were instructed to produce these strategies used them more often, which in turn benefited their recall. Another reason that testers may have benefited is that training involved two components of self-regulated study - both self-testing and its use in study allocation - that required a great deal of practice. In other words, metacognitive training may improve learning not only by increasing the overall use of these component skills across a list, but also by increasing the quality of their use. A practical implication is that screening for prior use of the targeted skills likely is unnecessary because even those who spontaneously self-tested or efficiently allocated study time benefited from training.

Although this metacognitive intervention was successful in improving memory performance, some potential limitations of this study should be mentioned. First, given a larger sample size, perhaps we would have detected significantly larger training gains in the non-tester group as compared to the tester group. Accordingly, our major conclusion here is that testers and non-testers can benefit from this metacognitive intervention, and not that the size of this benefit was identical for both groups. Second, by excluding older adults based on a history of dementia or other diagnosed memory problems, we cannot evaluate the generalizability of these training benefits 
to non-healthy adults. However, Camp [11] has demonstrated that adults diagnosed with dementia of the Alzheimer's type (DAT) can learn new information when the information is repeatedly tested across increasing intervals. Although the author demonstrated the benefits of using this spaced-retrieval technique, the DAT participants went through training sessions with a qualified practitioner, because the DAT participants would be unable to train themselves. Thus, although interventions based on self-testing - as in the current metacognitive intervention - can benefit older adults' learning, an athome version of this intervention may be mainly beneficial to older adults who are relatively high functioning.

Finally, the present intervention research focused exclusively on whether at-home training would benefit older adults' learning, as shown previously when experienced trainers conducted the intervention [3]. We did not expand the protocol to include either criterion tests that were delayed a great deal after training or those that were not explicitly trained in the manual; thus, we could not examine how long the trained skills were maintained or whether they would transfer to other tasks. Concerning the former, given how simple the component skills (selftesting and study allocation) are to understand and how easy they are to master, we suspect that the benefits of this intervention will be maintained over long intervals. If they are not maintained, a brief reminder to self-test and allocate appropriately may be enough for older adults to regain the benefits initially enjoyed after training. As for transfer, Dunlosky et al. [3] also included an untrained criterion task (self-paced study of individual words followed by free recall), and the benefits of the metacognitive intervention that focused on training paired-associate learning did not transfer to this free-recall task. Given that transfer may be most likely when training focuses on the broader applicability of the component skills across many contexts $[12,13]$, the metacognitive intervention may produce considerable transfer when the component metacognitive skills are practiced with multiple tasks during training. Importantly, these maintenance and transfer issues can be systematically explored using versions of the at-home training manual, which also makes it a valuable tool for training research.

Future research concerning metacognitive training also should assess the maintenance of training gains. In the present study, participants demonstrated significant gains in associative learning immediately following training; however, the long-term benefits of the metacognitive skills were not measured. Previous research has indicated that the likelihood of obtaining long-term benefits can be increased by offering supplemental attentional and relaxation training [14] or by offering booster training [2]. Moreover, recent evidence from the testing-effects literature suggests that metacognitive training could be even more effective if participants are taught to use a different allocation policy. The training program used here teaches older adults to self-test and then to drop from study those items that they can currently recall. Although dropping recalled items is efficient because more time is available for restudying unlearned items [15], this method may not optimize memory performance [16], because items that are recalled once still benefit from further testrestudy trials [17]. In fact, encouraging participants to drop items after only one correct recall could explain why metacognitive training has not always improved older adults' learning [18]. Thus, one possible means of enhancing the benefits of metacognitive training would be to encourage participants to correctly recall items more than once before removing them from further study.

In summary, metacognitive training administered with a take-home manual benefited older adults' learning, regardless of whether the adults reported using the strategy prior to training. This training technique can be easily combined with other effective mnemonic strategies that boost learning and hence shows much promise for promoting memory competence in late adulthood.

\section{Acknowledgement}

This research was supported by a grant from the National Institute on Aging, one of the National Institutes of Health (R37 AG13148).

References

1 Verhaeghen P, Marcoen A, Goossens L: Improving memory performance in the aged through mnemonic training: a meta-analytic study. Psychol Aging 1992;7:242-251.

-2 Ball K, Berch DB, Helmers KF, Jobe JB, Leveck MD, Marsiske M, Rebok GW, Smith DM, Tennstedt SL, Unverzagt FW, Willis SL: Effects of cognitive training interventions with older adults: a randomized controlled trial. JAMA 2002;288:2271-2281.

3 Dunlosky J, Kubat-Silman AK, Hertzog C: Training monitoring skills improves older adults' self-paced associative learning. Psychol Aging 2003; 18:340-345.

4 Woolverton M, Scogin F, Shackelford J, Black S, Duke L: Problem-targeted memory training for older adults. Neuropsychol Dev Cogn B Aging Neuropsychol Cogn 2001;8:241255 
5 West RL, Bagwell DK, Dark-Freudeman A: Self-efficacy and memory aging: the impact of a memory intervention based on self-efficacy. Neuropsychol Dev Cogn B Aging Neuropsychol Cogn 2008;15:302-329.

6 Price J, Hertzog C, Dunlosky J: Age-related differences in strategy knowledge updating: blocked testing produces greater improvements in metacognitive accuracy for younger than older adults. Neuropsychol Dev Cogn B Aging Neuropsychol Cogn 2008; 15:601626.

7 Dunlosky J, Hertzog C: Measuring strategy production during associative learning: the relative utility of concurrent versus retrospective reports. Mem Cognit 2001;29:247253.

8 Ekstrom RB, French JW, Harman HH, Dermen D: Manual for Kit of Factor-Referenced Cognitive Tests. Princeton, Educational Testing Service, 1976.
9 Salthouse TA, Babcock RL: Decomposing adult age differences in working memory. Dev Psych 1991;27:763-776.

10 Cohen J: Statistical Power Analysis for the Behavioral Sciences, ed 2. Hillsdale, Erlbaum, 1988.

11 Camp C: Facilitation of new learning in Alzheimer's disease; in Gilmore G, Whitehouse P, Wykle M (eds): Memory, Aging, and Dementia: Theory, Assessment, and Treatment. New York, Springer, 1989, pp 212-225.

12 Barnett SM, Ceci SJ: When and where do we apply what we learn? A taxonomy for far transfer. Psychol Bull 2002;128:612-637.

13 Verhaeghen P: The interplay of growth and decline: theoretical and empirical aspects of plasticity of intellectual and memory performance in normal old age; in Hill RD, Bäckman L, Stigsdotter Neely A (eds): Cognitive Rehabilitation in Old Age. New York, Oxford University Press, 2000, pp 3-22.
4 Stigsdotter-Neely A, Backman L: Multifactorial memory training with older adults: how to foster maintenance of improved performance. Gerontology 1989;35:260-267.

15 Pyc MA, Rawson KA: Examining the efficiency of schedules of distributed retrieval practice. Mem Cognit 2007;35:1917-1927.

16 Kornell N, Bjork RA: Optimising self-regulated study: the benefits and costs of dropping flashcards. Memory 2008;16:125-136.

17 Karpicke JD, Roediger HL III: Repeated retrieval during learning is the key to longterm retention. J Mem Lang 2007;57:151162.

18 Dunlosky J, Cavallini E, Roth H, McGuire CL, Vecchi T, Hertzog C: Do self-monitoring interventions improve older adult learning? J Gerontol B Psychol Sci Soc Sci 2007;62B: $70-76$. 